\title{
When do Aided Auditory Thresholds Reach the Speech Spectrum after Cochlear Implant Switch on?
}

\author{
Maria Rina T. Reyes-Quintos and Charlotte M. Chiong
}

Department of Otorhinolaryngology, College of Medicine and Philippine General Hospital, University of the Philippines Manila

\begin{abstract}
It is not easy to predict when the aided auditory thresholds of a child with cochlear implants will reach speech spectrum levels.

Objectives. This paper aims to determine the length of time before a cochlear implantee would reach aided hearing threshold of $\leq 45 \mathrm{dBHL}$ at 5 frequencies (target threshold) and whether there is a difference depending on age of implantation and sex.
\end{abstract}

Methods. From January 2009 to February 2011, the aided hearing thresholds of 41 prelingually deaf children with cochlear implants after switch on were reviewed.

Results. There were 21 males and 20 females. Majority of children $<4$ years old reached the target threshold gradually before the $12^{\text {th }}$ month of post-implantation; those who were 4-7 years old reached it within 3 months time while children $>7$ years old reached it at $\leq 1$ month. The probability that a female would reach the target threshold on or before 6 months was $90 \%$ but this was not statistically significant.

Conclusion. Sixty-six percent of the children with cochlear implants reached the target threshold within the first 3 months post fitting and $80 \%$ within the first 6 months post fitting. More of the older children reached target threshold earlier than the younger children. There was a trend that more females reached the target threshold earlier than the males but this was not statistically significant

Key Words: cochlear implants, auditory thresholds, cochlear implant fitting

\section{Introduction}

Cochlear implants are devices that are surgically placed inside the cochlea to aid children with severe to profound hearing losses, who derive minimal benefit from conventional hearing aids, to hear. The main goal is the development of normal speech and language in these children.

Corresponding author: Maria Rina T. Reyes-Quintos, MD

Department of Otorhinolaryngology

Philippine General Hospital

University of the Philippines Manila

Taft Avenue, Ermita, Manila 1000 Philippines

Telephone: +6325264360

Email: rinatrq@yahoo.com
When a pediatric cochlear implant patient (cochlear implantee) would begin to hear normal speech is often unpredictable. This information is important in order to reassure anxious patients and their parents. It is also useful for teachers, speech pathologists and audiologists because this will alert them to potential problems so that immediate steps could be taken to investigate and plan possible interventions as soon as possible. Factors that may affect outcomes are many, such as age, whether the implantee is pre or postlingual, and the history of previous hearing aid use.

After cochlear implantation and switch on of the device, evaluation materials and techniques to determine outcomes include speech and language evaluation tools which may be done in quiet or in noise, and aided hearing tests in quiet and in noise. The advantage of the aided hearing test in quiet is that it is easy to perform and the results are easy to compare longitudinally and horizontally. Additionally, the maximum hearing capability of the child is obtained under ideal conditions. When evaluating the aided hearing test, attention is paid to the tones within the speech spectrum which is usually presented as the "speech banana". The speech banana encompasses the frequencies and corresponding decibels required to hear speech from low to high frequencies. When hearing thresholds reach $45 \mathrm{~dB} \mathrm{HL}$, there is beginning access to speech sounds, particularly in the low and midfrequencies. Medline search shows that there have been no previous studies done on this topic. The consequence of reaching the speech spectrum may help in predicting speech and language outcomes in children.

This study aims to determine the time interval needed for the cochlear implantee to reach a hearing threshold of $\leq 45 \mathrm{dBHL}$ at 5 frequencies (target threshold) and whether sex and age of implantation has an impact.

\section{Methods}

The database of the cochlear implant program of the Department of Otorhinolaryngology of the University of the Philippines, Philippine General Hospital using the Medical Electronics (Med-El) implant device recorded 59 pediatric cochlear implantations from January 2009 to February 2011. Included in this study are forty one (41) congenitally deaf children (prelingual) who regularly had their cochlear implant fitting post switch on for at least one session every 2 
months for a minimum of 6 months by an audiologist (Table 1). Excluded were those with neurodevelopmental disorders such as the autism spectrum disorder and mental retardation and those with additional disabilities.

Table 1. Age and sex distribution of cochlear implant cases (prelingual), January 2009-February 2011.

\begin{tabular}{lccl}
\hline \multicolumn{1}{c}{ Age Range } & Male & Female & Total \\
\hline 1 to $<4$ years & 9 & 7 & 16 \\
4 to 7 years & 8 & 8 & 16 \\
$>7$ years & 4 & 5 & 9 \\
Total & 21 & 20 & 41 \\
\hline
\end{tabular}

All of the children underwent CT scan of the temporal bone. Majority wore hearing aids for at least 6 months (Table 2). However, the aided threshold data using their hearing aids were not available. One of the 2 children who did not use hearing aids prior to cochlear implantation was a 3 yearold male who had a history of rubella while the other was a 5 year-old with congenital hearing loss of unknown cause (Table 3). Hearing aids were prescribed to these children but they refused to wear them.

Table 2. Cause of deafness and use of hearing aid among cochlear implant cases, January 2009 - February 2011.

\begin{tabular}{|c|c|c|c|}
\hline \multirow[b]{2}{*}{ Cause of deafness: } & \multicolumn{2}{|c|}{$\begin{array}{l}\text { Hearing aid prior to } \\
\text { Cochlear Implant }\end{array}$} & \multirow{2}{*}{$\begin{array}{c}\text { Total } \\
\text { No. of } \\
\text { Cases }\end{array}$} \\
\hline & No & Yes & \\
\hline Congenital Rubella & 1 & 16 & 17 \\
\hline Large Vestibular Aqueduct & & 3 & 3 \\
\hline Ototoxic medications & & 4 & 4 \\
\hline \multicolumn{4}{|l|}{ (Prolonged ICU stay) } \\
\hline Meningitis & & 1 & 1 \\
\hline Auditory neuropathy & & 1 & 1 \\
\hline Undetermined & 1 & 14 & 15 \\
\hline Total & 2 & 39 & 41 \\
\hline
\end{tabular}

Table 3. Cause of deafness and sex of the cochlear implant cases (prelingual) January 2009 - February 2011.

\begin{tabular}{lccc}
\hline \multicolumn{1}{c}{ Cause of deafness } & Male & Female & Total \\
\hline Congenital rubella & 9 & 8 & 17 \\
Large Vestibular Aqueduct & 2 & 1 & 3 \\
Ototoxic Medications & 2 & 2 & 4 \\
Meningitis & 1 & 0 & 1 \\
Auditory neuropathy & 0 & 1 & 1 \\
Undetermined & 7 & 8 & 15 \\
Total & $\mathbf{2 1}$ & $\mathbf{2 0}$ & $\mathbf{4 1}$ \\
\hline
\end{tabular}

Switch on of the cochlear implant devices were done after 1 month to allow for stabilization and healing of the implant site. Follow-ups for cochlear implant device fitting and aided hearing thresholds were done every 2 weeks for the first 3 months, once a month for the next 3 months and every 6 months thereafter. However, patients were free to have fitting and aided test whenever they felt that there was a need, like before the school year starts or before a trip abroad.

During the switch on, the processor was connected to a computer and this communicates with the implanted device through a magnet sealed under the skin in the temporal area. Telemetry was initially done to determine the impedance of the electrodes. If there were any extracochlear electrodes, high impedance or short circuit, these electrodes were switched off. Fitting or mapping of the cochlear implant device was then performed. The goal of fitting is to determine their most comfortable loudness (MCL) since this is the setting wherein sounds are best heard. In children 0-3 years old, behavioral responses to increasing electrode currents were observed. The child may smile, cry or sometimes ignore the stimuli. Electrode current are maintained at a level that is deemed comfortable and not irritating or painful to the child. In children who ignore the sounds initially or are not cooperative, a soft (low current level) setting is programmed in the beginning. In older children, it is usually possible to set the program at a level more or less close to their MCL. Four programs are frequently provided at increasing levels. The parents were taught and advised on how to use the device. They were also advised to continue their speech and language therapy. During the succeeding visits, aided hearing thresholds via soundfield were performed with an audiometer (Aurical Plus Diagnostic Audiometer by distributed by Meditron) using warble tones for the frequencies $250 \mathrm{~Hz}, 500 \mathrm{~Hz}, 1$ $\mathrm{kHz}, 2 \mathrm{kHz}$ and $4 \mathrm{kHz}$. Visual response audiometry were done for children less than 2 years old, play audiometry for children 2 to 4 years old and older children are asked to raise their hand if warble sounds are detected. These results were recorded and analyzed. All children were evaluated and were undergoing speech and language rehabilitation with various private and charitable institutions. Unfortunately, not all their records were available.

\section{Results}

The data of 41 pediatric cochlear implantees (Med-El) from January 2009 to February 2011 were reviewed. Rubella was the most common cause of deafness. There were 20 males and 21 females. Sixteen patients were 1 to $<4$ years old; 16 were 4 to 7 years old and 9 were $>7$ years old. The aided test results were performed using age and developmentally appropriate techniques. Visual response audiometry were done for children less than 2 years old, play audiometry for children 2 to 4 years old and older children are asked to raise their hand if warble sounds are detected.

For females, the probability (relative risk) that their aided hearing threshold would reach the target threshold on or before 6 months post switch on was $90 \%$ while for males it was $70 \%$. However, this was not found to be statistically significant (Figure 1). 


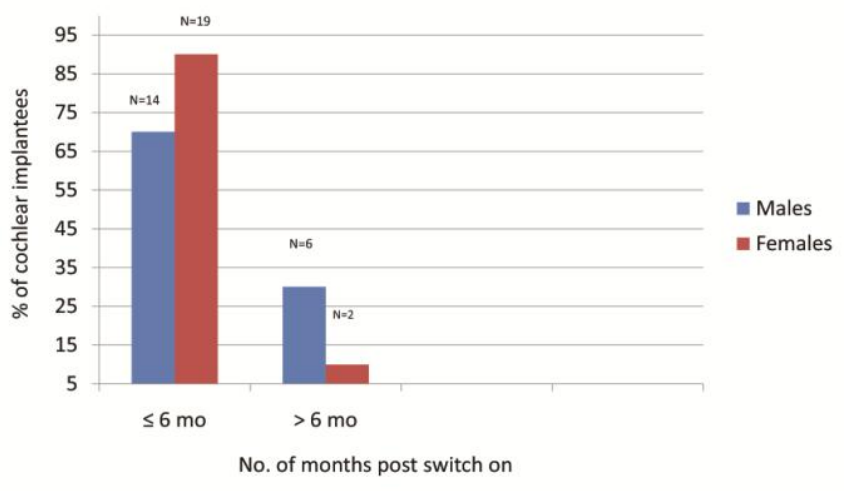

$\%$ of cochlear implantees reaching target threshold on or before 6 months and after 6 months in males and females.

$$
p=0.188
$$

Figure 1. Comparison of male and female children cochlear implantees who reached target threshold before 6 months and after 6 months.

All the children reached the target threshold by the $12^{\text {th }}$ month of post cochlear implantation. The older children reached the target threshold earlier. For those who were $<4$ years old, $50 \%$ reached the target threshold by 3 months post $\mathrm{s}$ witch on, while the other half continued to improve until they reached the target threshold before 12 months. For the children 4 to 7 years old, $75 \%$ reached the target threshold by 3 months post switch on and for those $>7$ years old, $70 \%$ reached the target threshold during the first month post switch on. When children $<4$ years old were compared with children 4 to 7 years old in attaining the target threshold, the probability of the former reaching the aforementioned threshold was 50\% compared to the latter which had the probability of $75 \%$ (Figure 2). However, when taken as a whole, $66 \%$ of the implantees reached $\leq 45 \mathrm{dBHL}$ threshold within the first 3 months post fitting and $80 \%$ within the first 6 months post fitting (Figure 3).

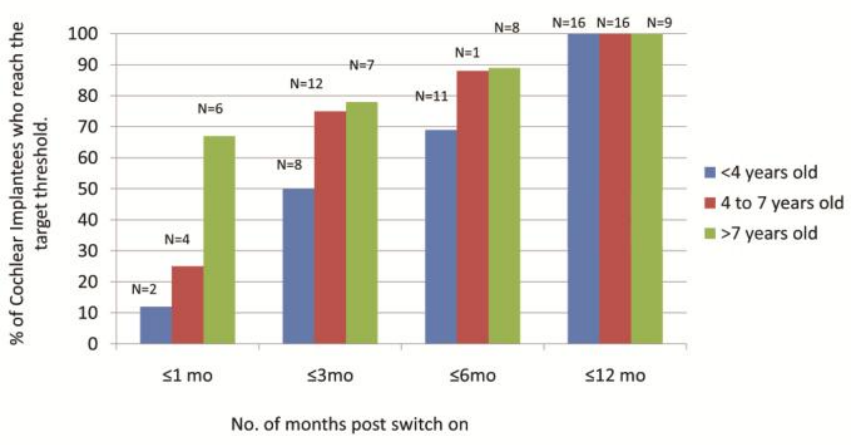

No. of months after switch on before aided thresholds reach target threshold in children $<4$ years old; $4-7$ years old and $>7$ years old

Figure 2. Cumulative number of months after switch on before target threshold is reached among children aged $<4$ years old; 4-7 years old and $>7$ years old.

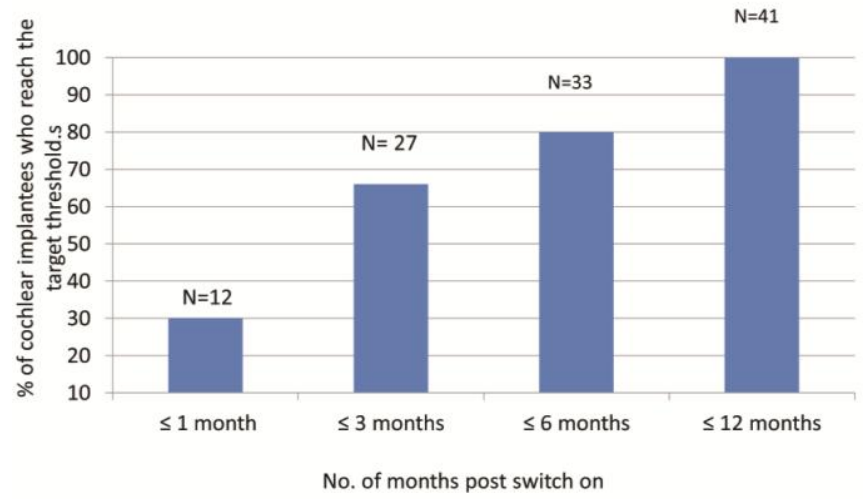

No. of months post switch on before target threshold is reached

Figure 3. Number of months post switch on before target threshold is reached by cochlear implantees.

\section{Discussion}

Studies have shown that there is a correlation between access to sound and development of speech. Children who have early access to sound (through hearing aids or cochlear implants) have an advantage of cortical plasticity which leads to rapid changes in the central auditory pathway often leading to normalization of the auditory cortex. ${ }^{1}$ Children implanted at less than 4 years old, began to develop age appropriate maturation of the central auditory system (there was a gradual change in the cortical evoked latency response) starting 3 months post switch on. The same was expected for most of the older implanted children if they had history of appropriate hearing aid use. ${ }^{2}$ In fact, it is interesting to note that in one study, pre-implantation rehabilitation using hearing aids in very young children does not have an impact on their language development after implantation ${ }^{3}$ probably because they were implanted during the critical period for language development. However, children implanted later are benefited by hearing aid use during this critical period before implantation wherein acoustic stimulation is vital for the development of speech and language.

Our study shows that in children $<4$ years old, more than half of the children with cochlear implants reached the target threshold within 3 months and the others continued to improve within the year. For the 4 to $<7$ years old group and the $>7$ years old group, they reached the $45 \mathrm{~dB}$ threshold earlier than the younger children, perhaps because they were easier to test audiologically, have had more exposure and experience with sound and longer speech and language therapy while still wearing hearing aids (before their implantation). Normalization of hearing thresholds in turn may translate to normal development of speech and language in children implanted early ${ }^{4}$ as well as implanted older children with history of appropriate hearing aid use.

The two children ( 3 and 5 years old, respectively) who did not use hearing aids before implantation reached the 
threshold in six and ten months. Although these are within the expected one year - it may indicate a slight delay toward normalization.

Why females would show a trend of earlier improvement in reaching the target threshold has not been extensively studied. This finding could be a consequence of earlier development in speech among female children that earlier studies have already shown. ${ }^{5}$ Indeed, males are also more likely to show speech disorders like problems in articulation and phonology. ${ }^{6}$ However, our study showed no statistically significant difference between males and females in the time interval before reaching the target threshold.

The results of our study may be used as guide in advising parents about what to expect with regard to speech and language after cochlear implantation especially in relation to the age and prior use of hearing aid. Additionally, a delay (e.g. A lack of improvement within 3-6 months in reaching the $45 \mathrm{~dB}$ threshold) should alert the concerned audiologist to delve into possible causes like misuse of the device, failure to attend therapy sessions and central auditory processing disorders. Appropriate interventions that include specialized tests (cortical evoked responses), change in speech and language strategies, and behavioural therapy may be needed.

Despite the aforementioned findings, there is still a need to correlate these results with the speech and language development of these children. The teaching style/method and home environment of these children as well as temperament of the child may also have some impact on how early they are able to reach the target threshold. An ongoing study in our institution on genetic markers may provide some basic answers to why some children reach the target threshold earlier than the others.

In conclusion, our findings show that young children implanted early and older children implanted with previous hearing aid use would usually reach the $\leq 45 \mathrm{~dB}$ aided hearing threshold within the first 3 months post implant.
Sixty six percent (66\%) of the children with cochlear implants reached the target threshold within the first 3 months post switch on and $80 \%$ within the first 6 months. Younger children showed gradual improvement within the first year while older children with previous hearing aid use reached the target threshold within 3-6 months. For females, the probability (relative risk) that their aided hearing threshold would reach the target threshold on or before 6 months post switch on was $90 \%$ while for males it was $70 \%$. However, this was not found to be statistically significant.

\section{Acknowledgment}

Many thanks to Dr. Erasmo Llanes for his invaluable help with the statistical analysis and Ms. Maria Luz San Agustin for making sure that our cochlear implant patients are tested properly, efficiently and with good humor at the Ear Unit of the Philippine General Hospital.

\section{References}

1. Gstoettner WK, Hamzavi J, Egelierler B, Baumgartner WD. Speech perception performance in prelingually deaf children with cochlear implants. Acta Otolaryngol. 2000; 120(2):209-13.

2. Sharma A, Nash AA., Dorman M. Cortical development, plasticity and re-organization in children with cochlear implants. J Commun Disord. 2009; 42(4):272-9.

3. Nicholas JG, Geers AE. Effects of early auditory experience on the spoken language of deaf children at 3 years of age. Ear Hear. 2006; 27(3):286-98.

4. Sharma A, Tobey E, Dorman M, et al. Central auditory maturation and babbling development in infants with cochlear implants. Arch Otolaryngol Head Neck Surg. 2004; 130(5):511-6.

5. Gender differences in the speech patterns of two and a half year old children [Online]. 1994 [cited 2011 Oct]. Available from http://www.assta.org/sst/SST-96/cache/SST-96-Chapter13-p15.pdf.

6. Incidence and prevalence of communication disorders and hearing loss in children [Online]. 2008 [cited 2011 Oct]. Available from http://www.asha.org/research/reports/children.htm. 